\title{
A NOVEL EFFICIENT HUMAN COMPUTER INTERFACE USING AN ELECTROOCULOGRAM
}

\author{
Vandhana ${ }^{1}$, Prabhu. $\mathrm{S}^{2}$ \\ ${ }^{1}$ PG Student, Sri Muthukumaran Institute of Technology \\ ${ }^{2}$ Assistant professor, Sri Muthukumaran Institute of Technology
}

\begin{abstract}
This paper presents the Electrooculography (EOG) on human computer interface $(H C I)$ is a novel measurement technique for eye tracking and the recognition for patients Eye movement and blood circulation in the nerves are measured by EOG signal. The EOG signal can be acquired by placing electrodes in the forehead and acquired data is analyzed by using wavelet transform and fuzzy logic. The transformed signal can be decomposed and de-noised. Fuzzy logic is used to separate the clustered signal from false signal. The analyzed signal can be given on the MSP430 controller which is implemented and used to control the electrical devices.
\end{abstract}

Keywords: Electrooculography (EOG), Human Computer interface (HCI), Multi signal processing (MSP), Eye movement

\section{INTRODUCTION}

Human computer interface is a field that promises to ease the communications between machines and humans. It is assumed that the population of people aged 60 and beyond will range from one to three in 2030 considering life span extension and the handicapped patients, they need for a human computer interface $(\mathrm{HCI})$ has been increasing cognitive functions are generally normal, patients with amyotrophic lateral sclerosis other tetraplegia clinical conditions have severe disabilities in moving their whole bodies. Some of these patients can only move their eyeballs. So by introducing a new channel without overt speaking and hand/arm motions makes life easier for patients and therefore improves their life style.

Paralyzed stroke patients are unable to normally communicate with their environment. Their body that is under their control, in terms of muscular movement using eyeballs [15]. As a review of the state of the art of electrooculogram (EOG) systems, there are several EOG based HCI applications for different purposes in the literature. Our motivation is to increase the quality of life of these patients using an HCI that provides an efficient communication channel.

The interface that provides control of machines for disabled people is called man machine interface (MMI) in general. When the control can be made by using a computer based or (microcomputer-based) system, it is called HCI, instead of MMI. The electrical signals generated by the human brain that are related to body functions are called an electroencephalogram (EEG). If the assistive system is based on EEG, it is known as the brain computer interface (BCI), and its applications for severely disabled people. The electrical signals generated by the eye movements which are called an electrooculogram (EOG).
The Electrooculogram (EOG) as a novel measurement technique for wearable eye tracking and recognition of user activity and attention in mobile settings. The EOG recordings to be implemented using electrodes integrated into glasses and the signals processed in real time on a light weight device worn on the body. This signal capturing eye wear system is used to project the graphical interface [3].

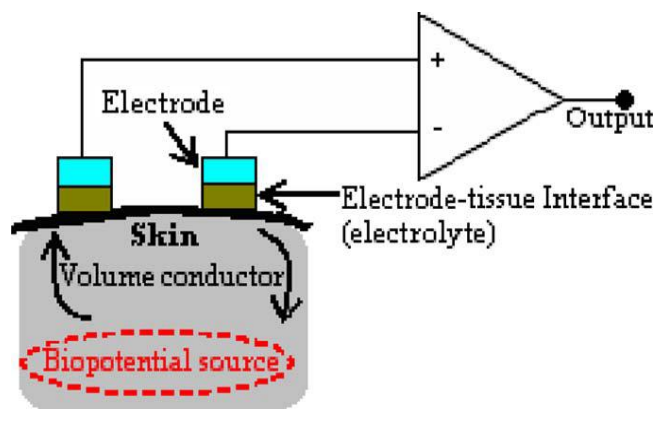

Fig 1.1 Electrode device

The fig1.1 shown the electrode circuit it can be capture the data or signal from the blood circulation on forehead on eye. The most efficient ways to acquire and analyze bioelectrical signals is through highly integrated software and hardware such as National Instruments SIGVIEW and data acquisition devices. SIGVIEW includes a powerful library with more than 500 functions for mathematics, signal processing, and analysis. Once the bioelectrical signal is amplified it can be digitized by any electrode data acquisition device, and then analyzed and displayed in SIGVIEW.

In recent technological advancements in human computer interface movement based interaction such as eye gaze 
tracking[1][8],track the pupil[13],Rapid Eye Movement (REM sleep)[14] and mobile gaze based[2]. The previous papers are used the EOG signal for control the wheel chair [8], virtual keyboard [4][7], remote devices[3], and eye controlled web browser[9]. They used to analyses the techniques are image processing algorithm, longest line scanning, cam shift algorithm, wavelet transform, neural network, Kanade optical flow algorithm, Clustering algorithm and Kalman filtering.

The existing system provides a HCI based on EOG signal, data analyzed in real time using a microcontroller based platform running the Linux operating system. The EOG signal is filtered using a CWT, here an eye movement is detected by using neural network.

The proposed project is analyzed the electrooculogram (EOG) signal by using wavelet transform and fuzzy logic. The EOG signal (Eye movement and blood circulation) can be acquired by placing the electrodes which is used in Human computer interface (HCI) it shown in Fig 1.2.

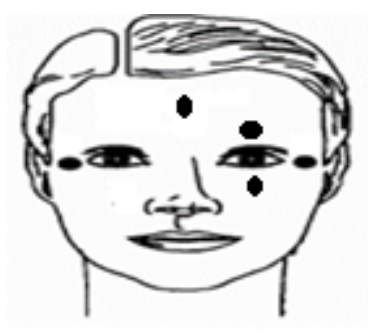

Fig 1.2 Positioning of electrodes

Horizontal-channel electrodes were placed on the right and left channel (Ch.H+ and Ch.H-), up and down channel (Ch.V+ and $\mathrm{Ch.V-)}$ this field focused on translating four eye movements (left, right,up, and down), and eye blink to select device which is vary from the pressure of the blood in the nerves. The EOG signal can be acquired from the electrode and the signal are amplified using the signal conditioning circuit. Then the acquired data is analyzed by using wavelet transform and fuzzy logic. The transformed signal can be decomposed and de- noised. Fuzzy logic is used to separate the clustered signal from false signal.

\section{SYSTEM DESGIN}

The block diagram describing the project is shown in Fig 2.1.The method consists of two steps namely 1 . Acquisition system 2.Control system

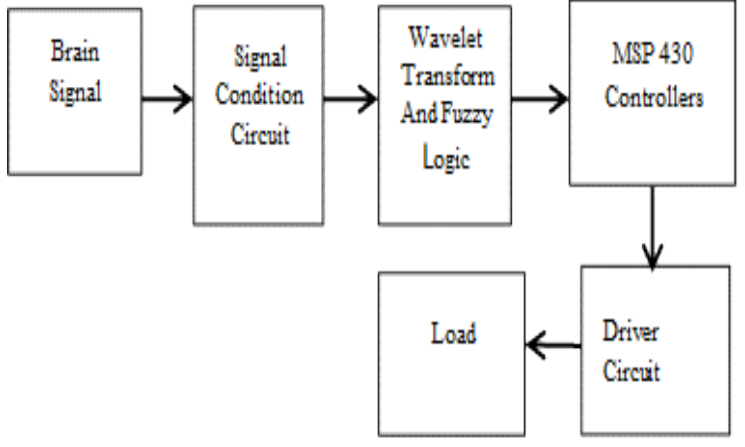

Fig 2.1 Block Diagram of Human Computer Interface

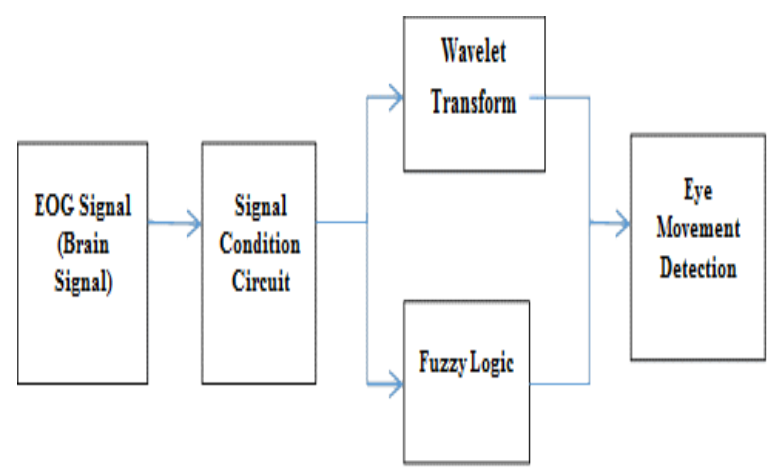

Fig 2.2 Block Diagram of Acquisition system

The electrode circuit, it can be capture the data or signal from the blood circulation on forehead on eye. The biomedical signals acquired from the human body are frequently very small. So that the signal can be amplified. The Wavelet Transform provides a time-frequency representation of the signal. Wavelet transform has ability to analysis different eye movement simultaneously in both time and frequency domain. Fuzzy Logic belongs to the family of many-valued logic, which focuses on fixed and approximate reasoning. A variable in fuzzy logic can take a value range from 0 and 1, which opposed to take true or false as in traditional binary sets. Fuzzy logic is used to separate the clustered signal from false signal and determine the value of true and false signal. The eye movement can be determined by using the wavelet transform and fuzzy logic. The digital wavelet transformed signal can be decomposed and de-noised. Then measure the range of de-noised. Thus the values are different for each movement.

\section{PHOTOS AND SPECIFICATION}

In the acquisition system, the 230v Dc power supply is given to transformer; it converts $230 \mathrm{v}$ to $12 \mathrm{v}$. Then is $12 \mathrm{v}$ power supply is given to bridge rectifier which converts Dc to Ac. now the $12 \mathrm{v}$ power supply is converted to $5 \mathrm{v}$ using voltage regulator. The $5 \mathrm{v}$ supply is given to signal conditioning circuit and this is amplifies the signal from the electrode. The signal obtain from the forehead will be from 58.12 to $68 \mathrm{mv}$, and then 
the amplified output will be $5 \mathrm{v}$. The amplified signal is the analog signal which is viewed in the SIGVIEW software.

\subsection{Acquisition System}

The block diagram describing the acquisition system, the brain signals are acquired using electrode, then the obtained signal are amplified and the signals are analyzed using wavelet transform and fuzzy logic. The detail block diagram is shown in the figure 2.2 .

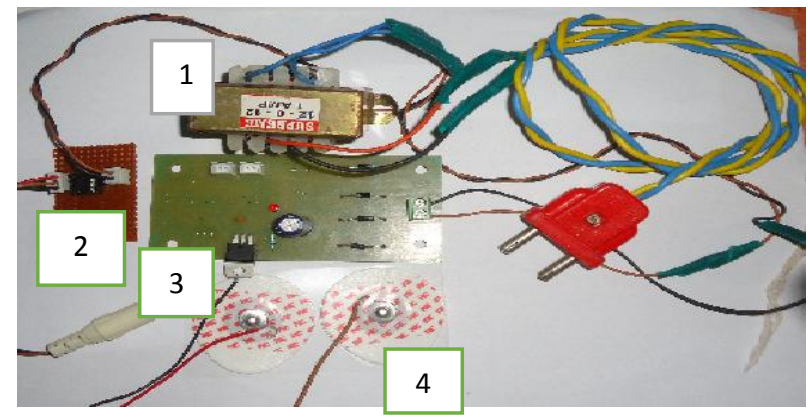

Fig 3.1 Hardware Component of Acquisition System

1. Transformer, 2. Signal conditioning, 3.Voltage regulator, 4.Electrode.

\section{RESULT AND DISCUSSION}

The simulation tool used is MATLAB13a. The MATLAB (matrix laboratory) is a numerical computing environment and fourth-generation programming language.

\subsection{Wavelet Transform}

The eye movement (signal) is analyzed in discrete Fourier transform, which is filtered and decomposed and it is denoised.

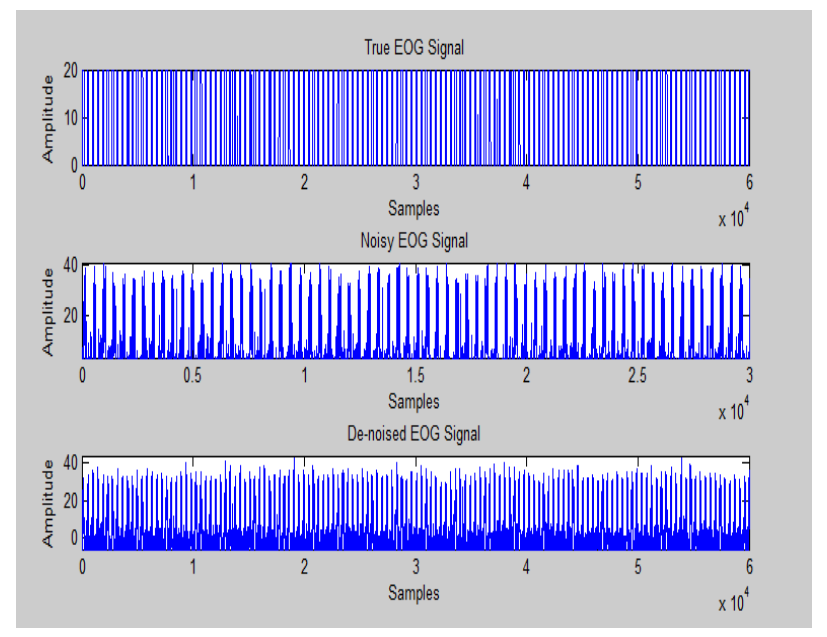

Fig 4.1 Noise and De Noised Signal for Left
The input signal of left movement is $20 \mathrm{khz}$ and it is analyzed using discrete Fourier transform and produces de-noised signal, then the output frequency of left eye movement will be $1.428 \mathrm{X} 10^{-3} \mathrm{HZ}$. Then the threshold value obtained 0.1596 .

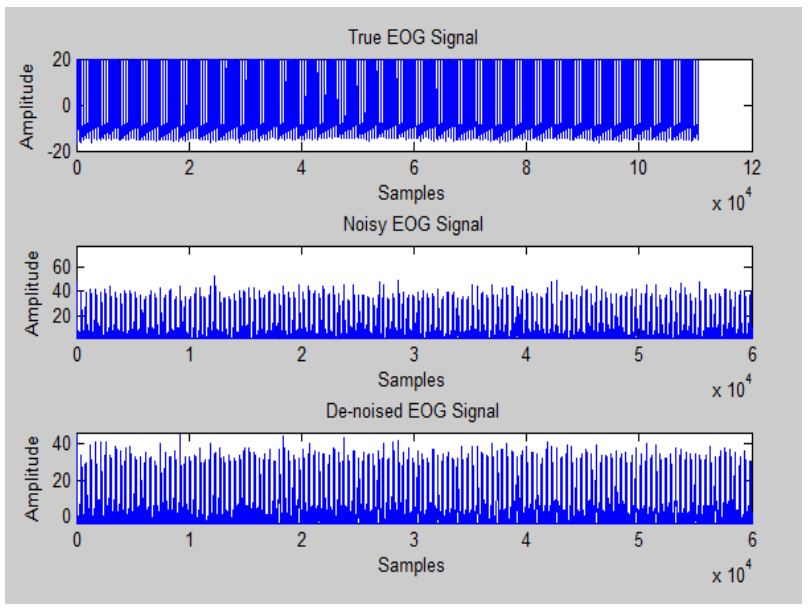

Fig 4.2 Noise and De Noised Signal for Right

The input signal of right movement is $23.9 \mathrm{khz}$ and it is analyzed using discrete Fourier transform and produces denoised signal, then the output frequency of right eye movement will be $1.25 \mathrm{X} 10^{-3} \mathrm{HZ}$. Then the threshold value obtained 0.1609 .

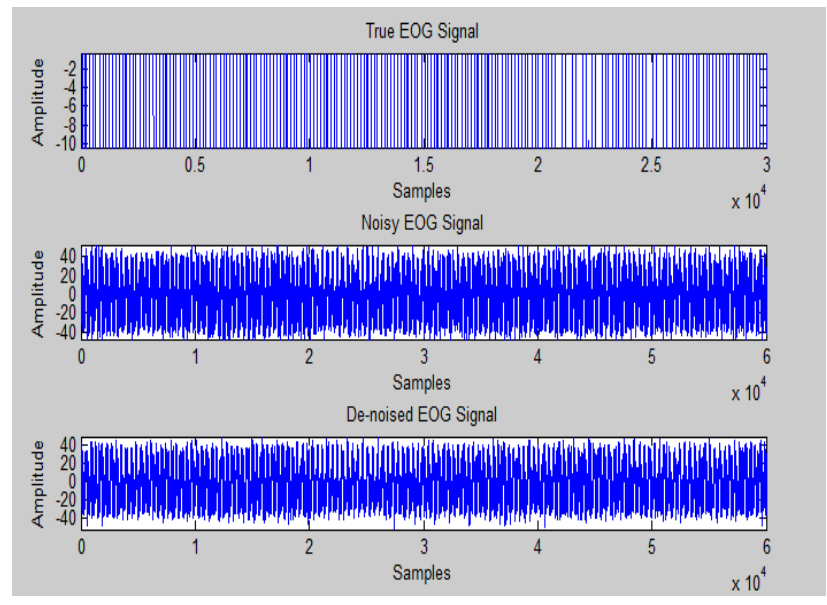

Fig 4.3 Noise and De Noised Signal for Upward

The input signal of up movement is $29 \mathrm{khz}$ and it is analyzed using discrete Fourier transform and produces de-noised signal, then the output frequency of up eye movement will be $1.0526 \mathrm{X} 10^{-3} \mathrm{HZ}$. Then the threshold value obtained 0.1152 . 


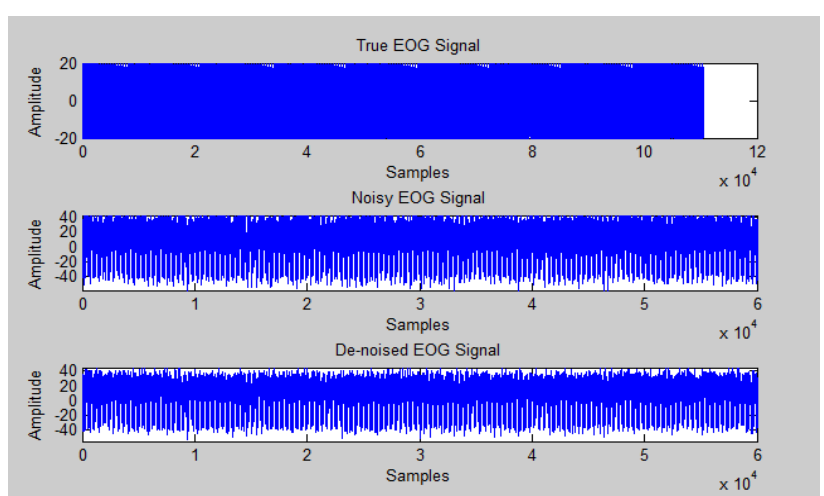

Fig 4.4 Noise and De Noised Signal for Downward

The input signal of upward movement is $25 \mathrm{khz}$ and it is analyzed using discrete Fourier transform and produces denoised signal, then the output frequency of left eye movement will be $1.538 \mathrm{X} 10^{-3} \mathrm{HZ}$. Then the threshold value obtained 0.1209 .

\section{THRESHOLD GRAPH}

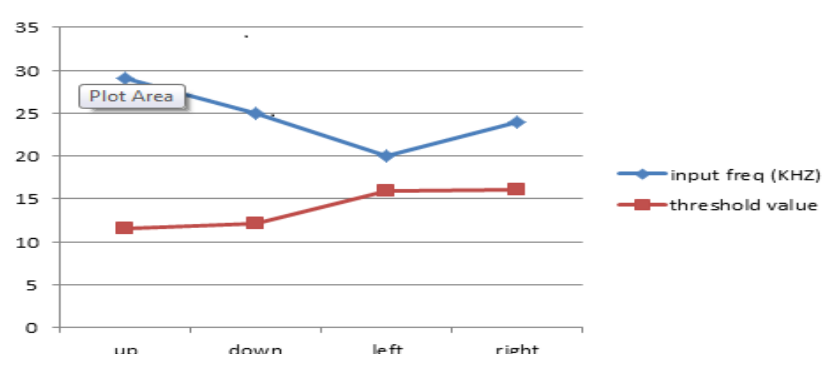

Fig 5.1 Graph of Frequency and Threshold

Figure 5.1 shows the threshold value and the frequency of different eye movements, where if there is any increase in input frequency, there could be a decrease in the respective threshold value.

\begin{tabular}{|l|c|c|}
\hline MOVEMENT & $\begin{array}{l}\text { INPUT } \\
\text { FRQUENCY } \\
\text { (KHz) }\end{array}$ & THRESHOLD \\
\hline Up & 29.0 & 0.1152 \\
\hline Down & 25.0 & 0.1209 \\
\hline Left & 20.0 & 0.1596 \\
\hline Right & 23.9 & 0.1609 \\
\hline
\end{tabular}

Fig 5.2 Comparative Table of threshold ranges for different eye movement

\section{FUZZY LOGIC}

The fuzzy logic is used to separate the true and false signal of different eye movement (left, right, up and down).

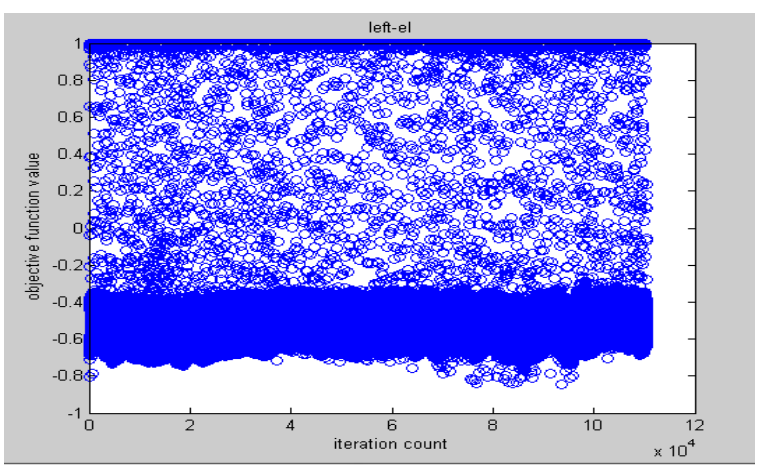

Fig 6.1 Output Of Clustered Signal For Left

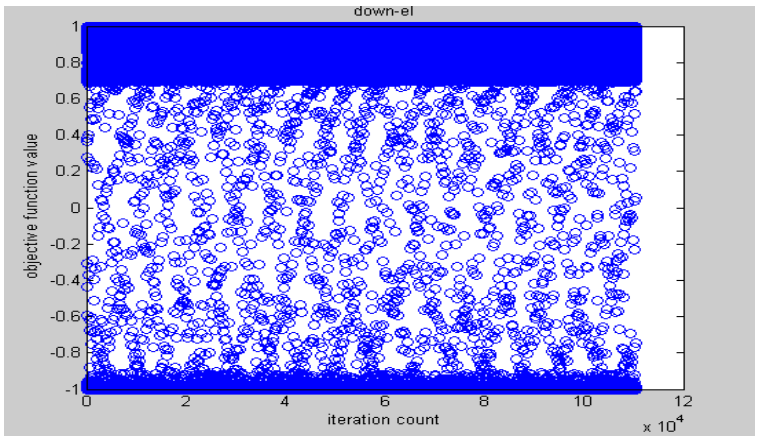

Fig 6.2 Output of Clustered Signal For Right

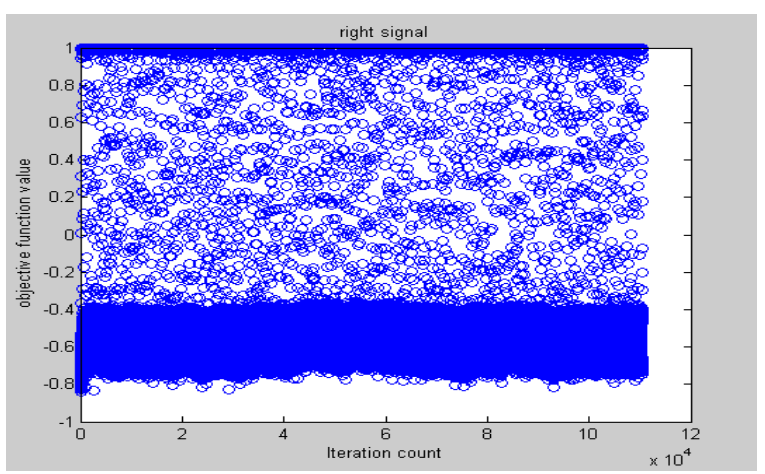

Fig 6.3 Output of Clustered Signal For Upward

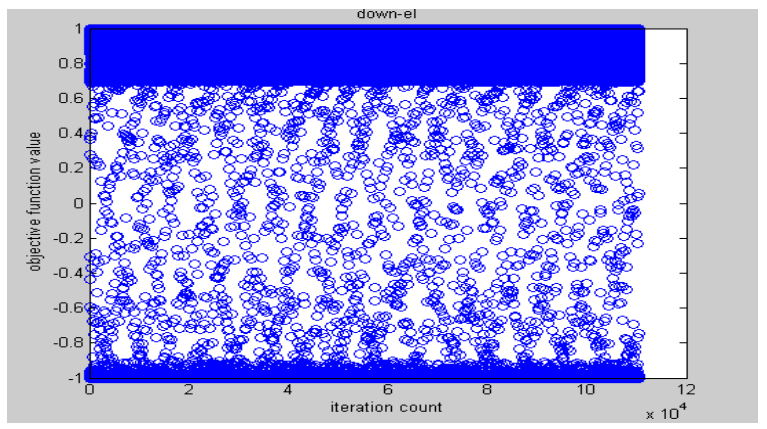

Fig 6.4 Output of Clustered Signal For Downward 


\begin{tabular}{|c|c|c|c|}
\hline EYE & TRUE & \multicolumn{2}{|c|}{ FALSE VALUE } \\
\cline { 3 - 4 } MOVEMENTS & VALUE & $\begin{array}{c}\text { Positive } \\
\text { region }\end{array}$ & $\begin{array}{c}\text { Negative } \\
\text { region }\end{array}$ \\
\hline LEFT & -0.3 to 0.9 & & -0.4 to - \\
& 0.9 to 1 & 0.7 \\
\hline RIGHT & -0.4 to 0.9 & $0.9-1$ & $\begin{array}{c}-0.4 \text { to }- \\
0.8\end{array}$ \\
\hline UPWARD & -0.5 to 0.9 & $0.9-1$ & 0.5 to -1 \\
\hline DOWNWARD & -0.9 to 0.7 & $0.7-1$ & -0.9 to -1 \\
\hline
\end{tabular}

Fig 6.5 comparative table for different eye movement

\section{CONCLUSIONS}

The EOG signal for different eye movements are classified in real time system in signal conditioning circuit and the quality of the graphic interface, for better controlling of the electrical devices.

The EOG signal analysis linear saccadic eye model and eye blinking, using wavelet transform and fuzzy logic in serial process and then it detects the corresponding eye movement. Then the captured EOG signal will be used for implementing algorithm and also for implementing the graphical user interface which controls electrical devices. Then by using MSP430 controller, human computer interface will be implemented.

\section{REFERENCES}

[1]. Ashlt talukder, john-michael morookian, s. Monacos, r. Lam, c. Lebaw, a. Bond, Real-time non-invasive eye tracking and gaze-point determination for human-computer interaction and biomedicine 2009

[2]. Andreas Bulling and Hans Gellersen, "Toward Mobile Eye-Based Human-Computer Interaction", Published by the IEEE CS n 1536-1268/10/\$26.00 @ 2010 IEEE

[3]. Andreas Bulling, Daniel Roggen and Gerhard Tröster,"Wearable EOG goggles:Seamless sensing and context-awareness in everydayenvironments",2009

[4]. Bulling A, Ward JA, Gellersen H, Tröster G," Eye movement analysis for activity recognition using electrooculography, IEEE Trans Pattern Anal Mach Intell, Apr;33(4):741-53. Doi: 10.1109/TPAMI.2010.86.

[5]. Barea.R , Boquete.L,Ortega.S, López.K, RodríguezAscariz.J," EOG-based eye movements codification for human computer interaction", Expert Systems with Applications 39 (2012) 2677-2683

[6]. Carlos a. Vinhais, fábio a. Santos, joaquim f. Oliveira, "An eog based human computer interface system for online control", CEMA 2010.

[7]. Damian Pakulski, Artur Gmerek, "The electrooculography control system", 2012

[8]. Heiko Drewes ," Eye Gaze Tracking for Human Computer Interaction", 2010
[9]. Królak.A, Strumiłło.P,"Eye-blink controlled humancomputer interface for the disabled", advances in intelligent and soft computing volume 60, 2009, pp 123-133 [10]. Kyung-nam kim and r. S. Ramakrishna, Vision-based eye-gaze tracking for human computer interface, 2006

[11]. Nurul Muthmainnah Mohd Noor, Salmiah Ahmad ,"Analysis of Different Level of EOG Signal from Eye Movement for Wheelchair Control,2009

[12]. Qiuping ding,kaiyu tong, and guang li, Development of an eog (electro-oulography)based human-computer interface, September 1-4, 2005

[13]. Subramanya amarnag, raghunandan s. Kumaran and john n. Gowdy, Real time eye tracking for human computer interfaces ,2007

[14]. Ruban.N, Suraj Kumar Panda, Swabhab Prakash Muduli, Mary Mekala,"Sleep Quality Monitor Using Stress Analysis and REM Sleep Detection", 20th August 2013. vol. 54 pp 2-4. [15]. Yash Shaileshkumar Desai," Natural Eye Movement and its application for paralyzed patients", International Journal of Engineering Trends and Technology, Volume4Issue4, 2013 